\title{
MicroRNA-28 promotes cell proliferation and invasion in gastric cancer via the PTEN/PI3K/AKT signalling pathway
}

\author{
LIHUA LI ${ }^{1,2}$, XIONGJIE ZHU ${ }^{1}$, TAO SHOU ${ }^{2}$, LIBO YANG $^{2}$, \\ XIAOZHEN CHENG ${ }^{1}$, JINTING WANG ${ }^{2}$, LIAN DENG ${ }^{1}$ and YANFANG ZHENG ${ }^{1}$ \\ ${ }^{1}$ Oncology Center, Zhujiang Hospital of Southern Medical University, Guangzhou, Guangdong 510282; \\ ${ }^{2}$ Department of Oncology, The First People's Hospital of Yunnan/Kunming University of Science \\ and Technology Affiliated Hospital, Kunming, Yunnan 650032, P.R. China
}

Received April 28, 2017; Accepted September 9, 2017

DOI: $10.3892 / \mathrm{mmr} .2017 .8299$

\begin{abstract}
Gastric cancer is the fourth most common malignant disease and second leading cause of cancer-associated mortalities worldwide. Previous studies revealed aberrantly expressed microRNAs (miRNAs) in various types of human cancer; these miRNAs play important roles in tumourigenesis and tumour development. miRNAs present a considerable potential for novel therapeutic approaches for treating human cancer. Therefore, the investigation of novel miRNAs involved in gastric cancer progression provides an opportunity to improve the prognosis of patients with gastric cancer. miRNA-28 (miR-28) has been investigated with regards to its expression and biological functions in many types of human cancer. However, previous studies have not discussed the expression patterns, roles and associated molecular mechanisms of miR-28 in gastric cancer. In the present study, miR-28 expression was identified to be upregulated in gastric cancer tissues and cell lines. miR-28 inhibition functionally inhibited cell proliferation and invasion in gastric cancer in vitro. Using bioinformatics analysis, luciferase reporter assay, reverse transcription-quantitative polymerase chain reaction and western blot analysis, phosphatase and tensin homolog (PTEN) was mechanically identified as a direct target of miR-28 in gastric cancer. PTEN was downregulated in gastric cancer and negatively correlated with miR-28 levels. Inhibition of PTEN restored the biological effects of miR-28 downregulation on the proliferation and invasion of gastric cancer cells. Notably, the downregulation of miR-28 results in the regulation of the phosphatidylinositol 3-kinase/protein kinase B signaling pathway in gastric cancer. These results suggested that miR-28
\end{abstract}

Correspondence to: Professor Yanfang Zheng, Oncology Center, Zhujiang Hospital of Southern Medical University, 253 Gongye Road, Guangzhou, Guangdong 510282, P.R. China

E-mail: 18665000236@163.com

Key words: microRNA-28, phosphatase and tensin homolog, gastric cancer, PI3K/AKT may be targeted for the development of novel treatments for gastric cancer in the future.

\section{Introduction}

Gastric cancer is the fourth most common malignant disease and second leading cause of cancer-related deaths worldwide (1). Approximately 850,000 newly diagnosed gastric cancer cases and 650000 deaths occur per year (2). Incidence and mortality of gastric cancer are the highest in East Asia (particularly in Korea, Mongolia, Japan and China); this disease became the second most lethal cancer in China (3). Major contributory risk factors to gastric cancer include Helicobacter pylori infection, dietary factors, tobacco use, alcohol consumption and obesity $(4,5)$. Despite considerable improvements in innovations in clinical diagnostics, surgical techniques and development of new chemotherapy regimens, five-year survival rates with advanced gastric cancer increased minimally in the past few years (6). Poor prognosis of gastric cancer patients mainly involves unlimited growth and strong metastatic capacities of gastric cancer cells $(7,8)$. Mechanism of gastric cancer oncogenesis remains largely unclear in spite of extensive clinical and basic research efforts $(9,10)$. Therefore, studies should focus on elucidating molecular mechanisms underlying gastric cancer occurrence and development and exploring novel therapeutic targets for gastric cancer treatments.

MicroRNAs (miRNAs) represent a large group of highly conserved and small RNA molecules of spanning 17-25 nucleotides (11). miRNAs regulate expression of their target genes in a post-transcriptional manner through interacting with 3'-untranslated regions (3'-UTRs) of targeted mRNA and causing mRNA degradation and suppression of translation (12). miRNAs regulate more than $60 \%$ of protein translation (13). Increasing studies demonstrated that miRNAs are aberrantly expressed in various types of human cancers and play important roles in tumorigenic processes, including cell proliferation, cycle, apoptosis, angiogenesis, invasion and metastasis $(14,15)$. Depending on their target genes, miRNAs may serve as either tumour suppressors or oncogenes (16). A large number of miRNAs contribute to gastric cancer tumourigenesis and tumour development by regulating 
expression of specific target genes; this condition suggests that miRNAs can be developed as therapeutic strategies for patients with gastric cancer (17).

miR-28 was studied with regard to its expression and biological functions in various types of human cancer (18-20). However, no previous research studied expression patterns, roles and associated molecular mechanisms of miR-28 in gastric cancer. This study detected expression levels of miR-28 in gastric cancer and determined its roles in regulation of aggressive behaviours of gastric cancer cells and its underlying mechanisms.

\section{Materials and methods}

Tissue samples and cell lines. A total of 31 paired gastric cancer tissues and adjacent normal gastric tissues were obtained from patients who had undergone radical gastrectomy at Zhujiang Hospital of Southern Medical University between January 2013 to December 2015. Normal adjacent tissues were collected at sites more than $4 \mathrm{~cm}$ away from the tumor margin. None of these gastric cancer patients had been treated with radiotherapy or chemotherapy before surgery. These tissues were immediately frozen in liquid nitrogen and then stored at $-80^{\circ} \mathrm{C}$. This study was approved by the Medical Ethics Committees of Zhujiang Hospital of Southern Medical University. Written informed consent was obtained from the patients enrolled in this research.

Five human gastric cancer cell lines (SGC-7901, MGC-803, MKN-1, BGC-823, AGS) and the normal gastric epithelium GES-1 cell line were all bought from the American Type Culture Collection (ATCC; Rockville, MD, USA). Cells were cultured in Dulbecco's modified Eagle's medium (DMEM) containing $10 \%$ fetal bovine serum (FBS) (both from Gibco; Thermo Fisher Scientific, Inc., Waltham, MA, USA), 100 U/ml penicillin and $100 \mu \mathrm{g} / \mathrm{ml}$ streptomycin in a humidified atmosphere with $5 \% \mathrm{CO}_{2}$ at $37^{\circ} \mathrm{C}$.

Cell transfection. The miR-28 inhibitor and corresponding scramble miRNA inhibitor negative control (NC inhibitor) were purchased from GenePharma (Shanghai, China). Small interference RNA (siRNA) targeting PTEN (PTEN siRNA) and non-target control siRNA (NC siRNA) were synthesized by Guangzhou RiboBio Co., Ltd. (Guangzhou, China). Cells were seeded into 6-well plates at a density of $6 \times 10^{5}$ each well. Cell transfection was performed when the cell density reached a confluence of $90 \%$. Cells were transfected with miR-28 inhibitor, NC inhibitor, phosphatase and tensin homolog (PTEN) siRNA or NC siRNA using Lipofectamine 2000 (Invitrogen; Thermo Fisher Scientific, Inc.) following to the manufacturer's instructions. Culture medium was replaced with fresh medium containing $10 \%$ FBS at $6 \mathrm{~h}$ post-transfection.

$R N A$ isolation and reverse transcription-quantitative polymerase chain reaction ( $R T-q P C R)$. Total RNA was extracted from gastric cancer tissue samples or cells using TRIzol reagent (Ambion; Thermo Fisher Scientific, Inc.), according to the manufacturer's protocol. The concentration and quality of the total RNA was evaluated using the ND-2000 spectrophotometer (NanoDrop Technologies; Thermo Fisher Scientific,
Inc., Wilmington, DE, USA). For miR-28 expression, cDNA synthesis was performed with TaqMan ${ }^{\circledR}$ MicroRNA Reverse Transcription kit (Applied Biosystems; Thermo Fisher Scientific, Inc.). Quantitative real-time PCR was conducted using the TaqMan MicroRNA Assay kit (Applied Biosystems; Thermo Fisher Scientific, Inc.). Relative expression level of miR-28 was normalized by U6 expression. For PTEN mRNA expression, reverse transcription was performed using PrimeScript RT Reagent kit (Takara Biotechnology Co., Ltd., Dalian, China). SYBR Premix Ex Taq Master Mix (Takara Biotechnology Co., Ltd.) was utilized to detect PTEN mRNA expression levels. GAPDH was used as an internal control for PTEN mRNA level. The relative expression was calculated using the $2^{-\Delta \Delta C t}$ method (21).

Cell Counting Kit 8 (CCK8) assay. Cell proliferation was determined using CCK8 assay according to the manufacturer's instructions. Briefly, transfected cells were collected and seeded into 96 -well plates $\left(3 \times 10^{3}\right.$ cells/well). 0, 24, 48, and 72 after incubation, cell proliferation was measured by the addition of $10 \mu \mathrm{l}$ of CCK8 solution (Dojindo Molecular Technologies, Kumamoto, Japan) into each well. After incubation at $37^{\circ} \mathrm{C}$ for $2 \mathrm{~h}$, absorbance was measured at a wavelength of $450 \mathrm{~nm}$ using a microplate reader (SpectraMAX Plus; Molecular Devices, LLC, Sunnyvale, CA, USA). The assays were performed in triplicates and repeated three times.

Matrigel invasion assay. Matrigel invasion assay was performed using 24-well Transwell chambers (8-mm pore size; EMD Millipore, Billerica, MA, USA) coated with Matrigel (BD Biosciences, Franklin Lakes, NJ, USA). In briefly, transfected cells were collected at $48 \mathrm{~h}$ posttransfection and suspended in DMEM without FBS. Transfected cells $\left(5 \times 10^{4}\right)$ were added to the top chamber, while the DMEM supplemented with $10 \%$ FBS used as a chemoattractant was added in the lower chamber. After $24 \mathrm{~h}$ incubation at $37^{\circ} \mathrm{C}$ with $5 \% \mathrm{CO}_{2}$, non-invasive cells on the top chambers were removed using cotton swabs. The invasive cells were fixed with methanol and stained with $0.1 \%$ crystal violet. Subsequent to washing three times with PBS, invasive cells in five randomly selected visual fields were photographed and counted under an inverted microscope (IX71; Olympus Corporation, Tokyo, Japan).

Bioinformatic analysis and luciferase reporter assay. Bioinformatic analysis was performed to predicate the potential targets of miR-28 with TargetScan (http://www.targetscan. org/) and PicTar (http://pictar.mdcberlin.de/).

The pGL3-wild type-PTEN-3'-UTR (pGL3-Wt-PTEN3'-UTR) containing the putative binding site of miR-28 and pGL3-mutant-PTEN-3'-UTR (pGL3-Mut-PTEN-3'-UTR) were chemically synthesized and obtained from GenePharma. For luciferase reporter assay, the plasmid (pGL3-Wt-PTEN-3'-UTR or pGL3-Mut-PTEN-3'-UTR) together with miR-28 inhibitor or NC inhibitor were transfected into cells using Lipofectamine 2000 reagent, according to the manufacturer's instructions. Luciferase activities were measured 48 h later with a dual-luciferase reporter system (Promega Corporation, Madison, WI, USA). Renilla luciferase activity was used for normalization. 
Western blot analysis. Tissue samples or cells were lysed using a radioimmunoprecipitation assay buffer (Beyotime Institute of Biotechnology, Haimen, China) in the presence of a protease inhibitor cocktail (Sigma-Aldrich; Merck KGaA, Darmstadt, Germany). A bicinchoninic acid protein assay kit (Beyotime Institute of Biotechnology) was used to detect protein concentration. Equal amounts of protein were separated by $10 \%$ sodium dodecyl sulfate-polyacrylamide gel electrophoresis and transferred to polyvinylidene fluoride membrane (EMD Millipore). After blocking with 5\% non-fat milk at room temperature in TBS, the membranes were incubated overnight at $4^{\circ} \mathrm{C}$ with specific primary antibodies for PTEN antibody (sc-133197; 1:1,000 dilution) and GAPDH antibody (sc-47724; 1:1,000 dilution) (both from Santa Cruz Biotechnology, Inc., Dallas, TX, USA). Subsequent to washing three times with TBST, the membranes were incubated with goat anti-mouse horseradish peroxidase-conjugated secondary antibody (sc-2005; 1:5,000 dilution; Santa Cruz Biotechnology, Inc.) at room temperature for $1 \mathrm{~h}$. Positive signals were developed by enhanced chemiluminescence solution (ECL; Pierce; Thermo Fisher Scientific, Inc.) and analyzed with ImageJ 1.49 (National Institutes of Health, Bethesda, MD, USA).

Statistical analysis. Data are presented as the mean \pm standard deviation, and compared with Student's t-test or one-way analysis of variance (ANOVA) using SPSS 18.0 (SPSS, Inc., Chicago, IL, USA). Correlation of miR-28 expression with that of PTEN mRNA was conducted with the Spearman's correlation analysis. Two-tailed p-value $<0.05$ was considered to indicate a statistically significant difference.

\section{Results}

miR-28 is upregulated in gastric cancer tissues and cell lines. To elucidate whether miR-28 correlates with progression of gastric cancer, its expression levels in 31 paired gastric cancer tissues and adjacent normal gastric tissues were determined using RT-qPCR. Data showed that miR-28 expression was upregulated in gastric cancer tissues compared with that in adjacent normal gastric tissues (Fig. 1A, P<0.05). Expression of miR-28 in a normal gastric cell line (GES-1) was compared with those of a panel of gastric cancer cell lines (SGC-7901, MGC-803, MKN-1,BGC-823 and AGS); and gastric cancer cell lines showed generally increased miR-28 expression (Fig. 1B, $\mathrm{P}<0.05)$. These results suggested that upregulation of miR-28 is a common event in gastric cancer and may play essential roles in gastric cancer progression.

Downregulation of miR-28 inhibits cell proliferation and invasion in gastric cancer. To investigate biological roles of miR-28 in development and progression of gastric cancer, miR-28 inhibitor was transfected into SGC-7901 and BGC-823 cells. RT-qPCR confirmed significant downregulation of miR-28 in SGC-7901 and BGC-823 cells after transfection with miR-28 inhibitor (Fig. 2A, $\mathrm{P}<0.05$ ). Based on CCK8 assay, miR-28 inhibitor transfection suppressed proliferation of SGC-7901 and BGC-823 cells compared with NC inhibitor group (Fig. 2B, $\mathrm{P}<0.05$ ). Matrigel invasion assay revealed that miR-28 knockdown decreased invasion abilities of SGC-7901 and BGC-823 cells (Fig. 2C, P<0.05). These results
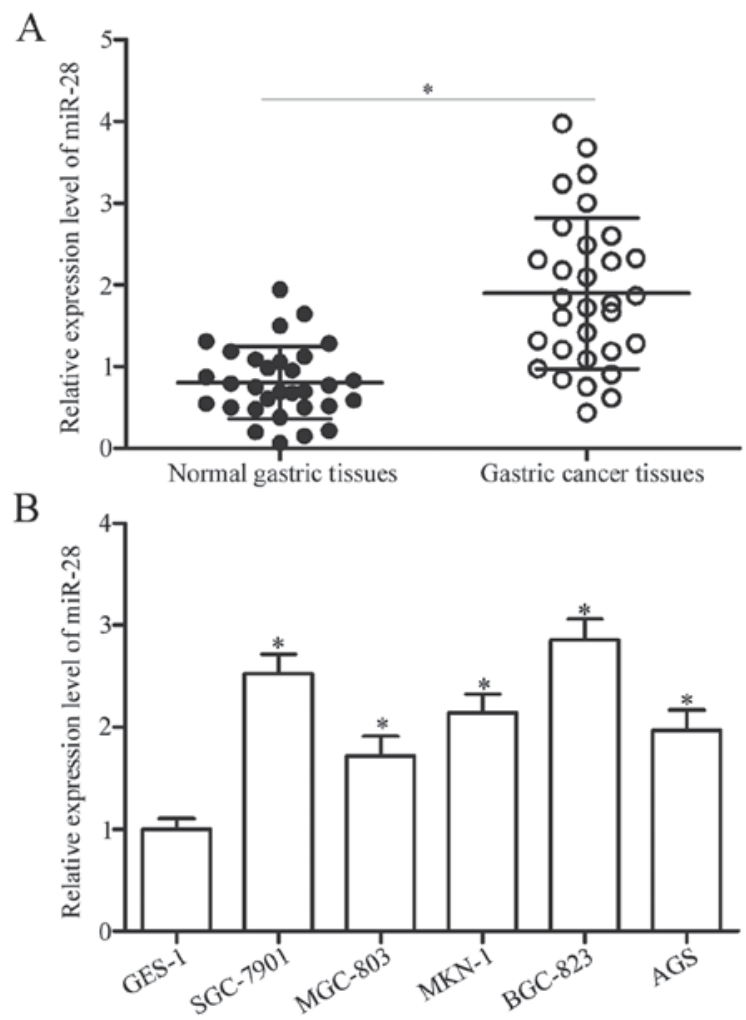

Figure 1. miR-28 is upregulated in gastric cancer tissues and cell lines. (A) Gastric cancer tissues exhibited higher expression levels of miR-28 in comparison with adjacent normal gastric tissues. ${ }^{*} \mathrm{P}<0.05$ compared with adjacent normal gastric tissues. (B) Using RT-qPCR, miR-28 expression was detected in gastric cancer cell lines (SGC-7901, MGC-803, MKN-1, BGC-823 and AGS) and a normal gastric cell line (GES-1). ${ }^{*} \mathrm{P}<0.05$ compared with GES-1. miR-28, miRNA-28.

demonstrated that miR-28 may serve as an oncogene in gastric cancer.

PTEN is a direct target of $m i R-28$. To elucidate underlying molecular mechanisms of miR-28 in gastric cancer, potential targets of miR-28 were predicted using bioinformatics analysis. Among candidates, PTEN was selected for further validation because it was downregulated in gastric cancer and participate in gastric cancer progression (22-24). Fig. 3A shows putative target sites of miR-28 in 3'-UTR of PTEN. To confirm whether miR-28 directly targets the 3'-UTR of PTEN, luciferase reporter assays were performed in SGC-7901 and BGC-823 cells transfected with plasmids (pGL3-Wt-PTEN-3'-UTR or pGL3-Mut-PTEN-3'-UTR) along with miR-28 inhibitor or $\mathrm{NC}$ inhibitor. Results showed that downregulation of miR-28 increased relative luciferase activities of wild-type PTEN 3'-UTR (Fig. 3B, P<0.05), whereas luciferase activities of mutant PTEN 3'-UTR remained unchanged.

mRNA and protein levels of PTEN levels in SGC-7901 and BGC-823 cells transfected with miR-28 inhibitor or NC inhibitor were examined to confirm regulatory roles of miR-28 in PTEN in gastric cancer. As shown in Fig. 3C and D, transfection of miR-28 inhibitor into SGC-7901 and BGC-823 cells resulted in remarkable upregulation of PTEN expression at both mRNA and protein levels $(\mathrm{P}<0.05)$. Overall, these results demonstrated that PTEN is a direct target gene of miR-28 in gastric cancer. 

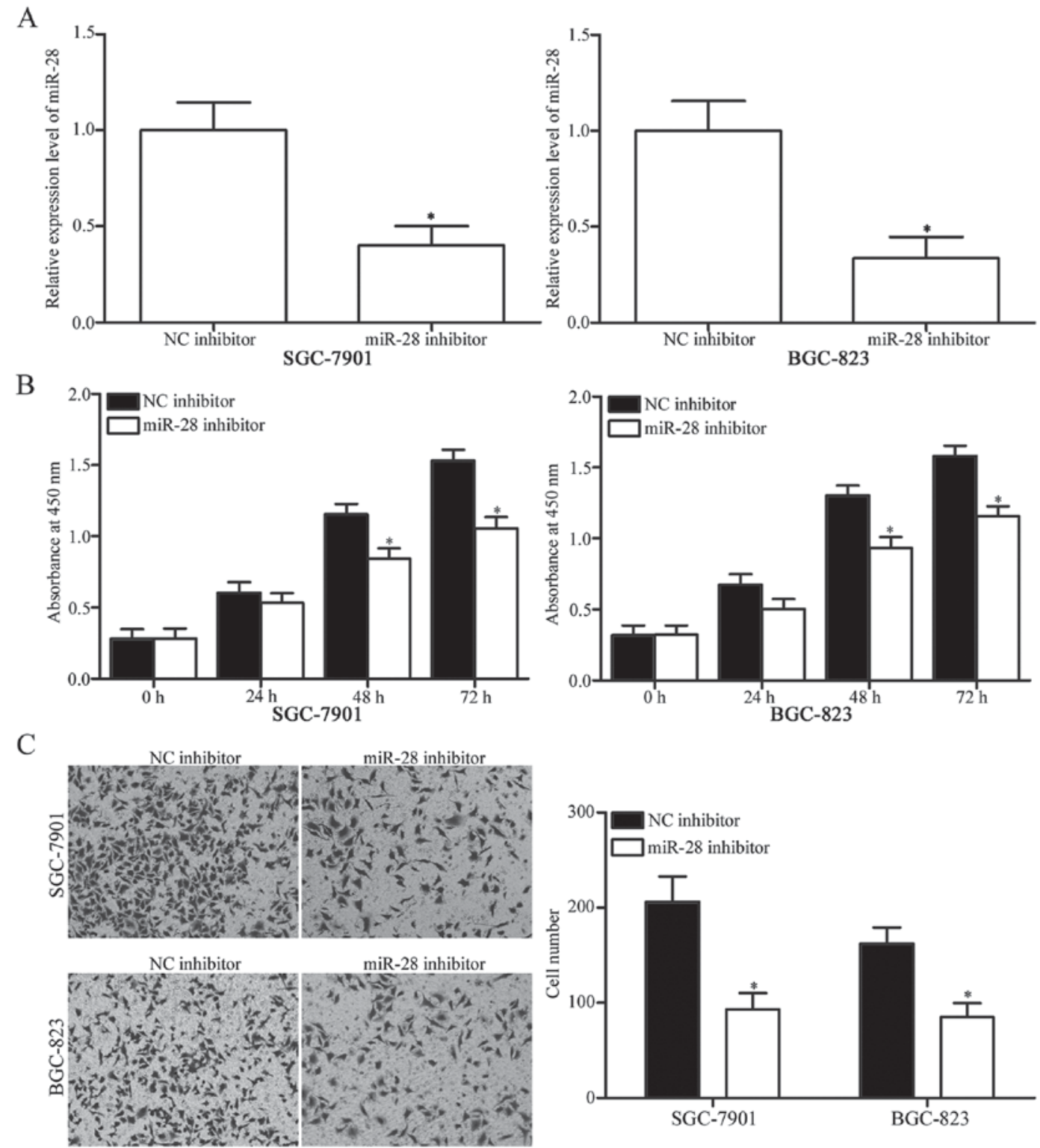

Figure 2. Downregulation of miR-28 inhibits proliferation and invasion of SGC-7901 and BGC-823 cells. (A) RT-qPCR analysis of miR-28 expression in SGC-7901 and BGC-823 cells after transfection with miR-28 inhibitor or NC inhibitor. ${ }^{*} \mathrm{P}<0.05$ compared with NC inhibitor. (B) CCK8 assay was used to evaluate proliferation of SGC-7901 and BGC-823 cells after transfection with miR-28 inhibitor or NC inhibitor. *P<0.05 compared with NC inhibitor. (C) Matrigel invasion assay was performed to investigate invasion capacities of SGC-7901 and BGC-823 cells transfected with miR-28 inhibitor or NC-inhibitor. "P<0.05 compared with NC inhibitor. miR-28, miRNA-28; CCK8, Cell Counting Kit 8.

An inverse correlation exists between PTEN and miR-28 in gastric cancer tissues. Subsequent examinations detected PTEN expression in gastric cancer tissues and adjacent normal gastric tissues. RT-qPCR analysis revealed upregulated expression of PTEN mRNA in gastric cancer tissues in comparison with adjacent normal gastric tissues (Fig. 4A, $\mathrm{P}<0.05$ ). Spearman's correlation analysis was used to evaluate association between PTEN mRNA and miR-28. As shown in Fig. 4B, miR-28 was strongly correlated with PTEN mRNA expression in gastric cancer specimens $(\mathrm{r}=-0.7264, \mathrm{P}<0.001)$, suggesting that upregulation of miR-28 in gastric cancer may primarily cause downregulation of PTEN.

PTEN knockdown restores functional effects of miR-28 on cell proliferation and invasion in gastric cancer. Rescue experiments were performed to evaluate whether PTEN is responsible for functional roles of miR-28 in gastric cancer cells. Firstly, SGC-7901 and BGC-823 cells were transfected with miR-28 inhibitor with or without PTEN siRNA. After transfection, Western blot analysis confirmed that PTEN expression was recovered in miR-28 inhibitor-transfected SGC-7901 and BGC-823 cells after transfection with PTEN siRNA (Fig. 5A, P<0.05). Subsequently, CCK8 assay and Matrigel invasion assays demonstrated that co-transfection of PTEN siRNA restored functional effects of miR-28 inhibitor on gastric cancer cell proliferation (Fig. 5B, $\mathrm{P}<0.05$ ) and invasion (Fig. 5C, P<0.05) in SGC-7901 and BGC-823 cells. These results demonstrated that miR-28 plays oncogenic roles in gastric cancer, at least in part, by targeting PTEN.

miR-28 inhibitor regulates PTEN/PI3K/AKT signalling in gastric cancer. Previous studies reported that PTEN is 
A

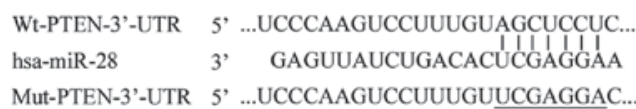

C

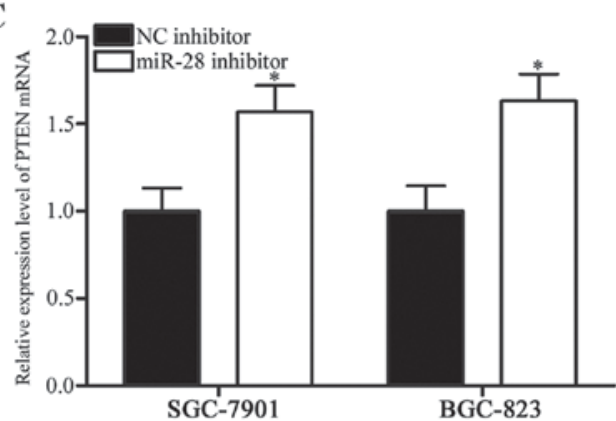

B

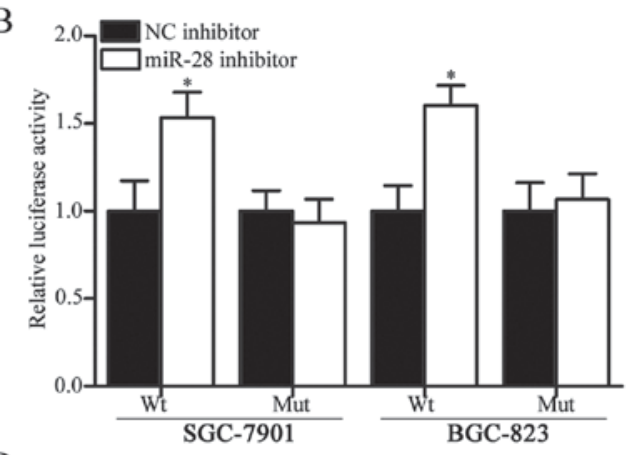

D
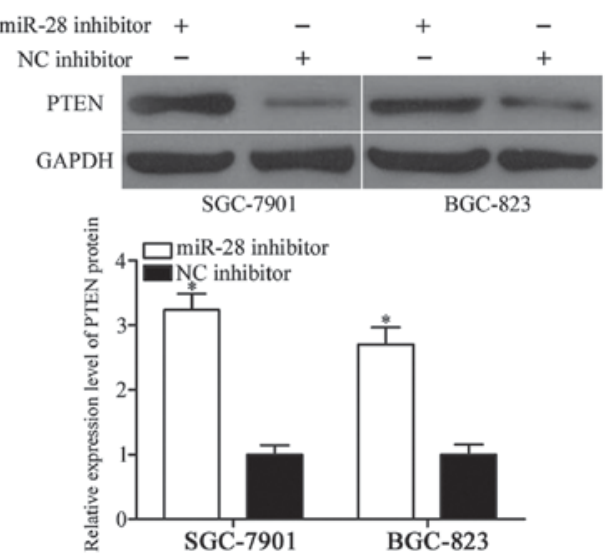

Figure 3. PTEN is a direct target of miR-28 in gastric cancer. (A) Putative binding sites for miR-28 in the 3'-UTR of PTEN were predicted using TargetScan and PicTar. The figure also shows mutated sites within binding sites of $3^{\prime}$-UTR of PTEN. (B) Downregulation of miR-28 increased luciferase activities of pGL3-Wt-PTEN-3'-UTR, whereas alteration of miR-28 featured no effect on luciferase activity of pGL3-Mut-PTEN-3'-UTR in both SGC-7901 and BGC-823 cells. " $\mathrm{P}<0.05$ compared with NC inhibitor. (C) RT-qPCR was used to evaluate effects of miR-28 knockdown on PTEN mRNA expression in SGC-7901 and BGC-823 cells. "P<0.05 compared with NC inhibitor. (D) Western blot analysis determined PTEN protein expression in SGC-7901 and BGC-823 cells treated with miR-28 inhibitor or NC inhibitor. ${ }^{*} \mathrm{P}<0.05$ compared with NC inhibitor. PTEN, phosphatase and tensin homolog; miR-28, miRNA-28; 3'-UTR, 3'-untranslated region.

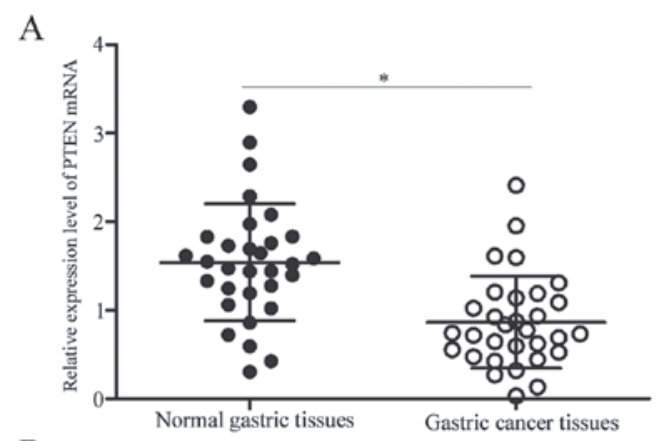

$\mathrm{B}$

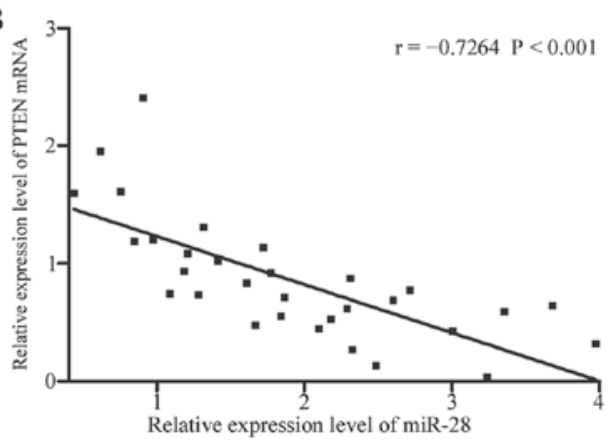

Figure 4. PTEN expression is inversely correlated with miR-28 expression levels in gastric cancer tissues. (A) Expression of PTEN mRNA was downregulated in gastric cancer tissues compared with adjacent non-tumour tissues. " $\mathrm{P}<0.05$ compared with adjacent non-tumour tissues. (B) Spearman's correlation analysis indicated an inverse correlation between PTEN mRNA and miR-28 expression in gastric cancer tissues. PTEN, phosphatase and tensin homolog; miR-28, miRNA-28. a master negative regulator of the PI3K/AKT signalling pathway in gastric cancer $(25,26)$. Hence, PI3K, AKT and p-AKT expression levels in SGC-7901 and BGC-823 cells were measured after transfection with miR-28 inhibitor or $\mathrm{NC}$ inhibitor. Results revealed that downregulation of miR-28 decreased protein levels of PI3K and p-AKT (Fig. 6, P<0.01) in SGC-7901 and BGC-823 cells but did not affect total AKT expression. These results suggested that miR-28 inhibits gastric cancer progression by regulation of PTEN/PI3K/AKT signalling pathway.

\section{Discussion}

Previous studies demonstrated aberrantly expressed miRNAs in various types of human cancers, and these miRNAs play important roles in tumourigenesis and tumour development $(27,28)$. miRNAs feature considerable potential for novel therapeutic approaches for treating human cancers (29). Therefore, investigation of novel miRNAs involved in gastric cancer progression provides opportunities to improve prognosis of gastric cancer patients. In this study, miR-28 expression was upregulated in gastric cancer specimens and cell lines. Downregulation of miR-28 inhibited gastric cancer cell proliferation and invasion through regulation of PTEN/PI3K/AKT signalling pathway. These results suggested that miR-28 plays a crucial role in gastric cancer and may be developed as a therapeutic target for patients with such disease. 
A

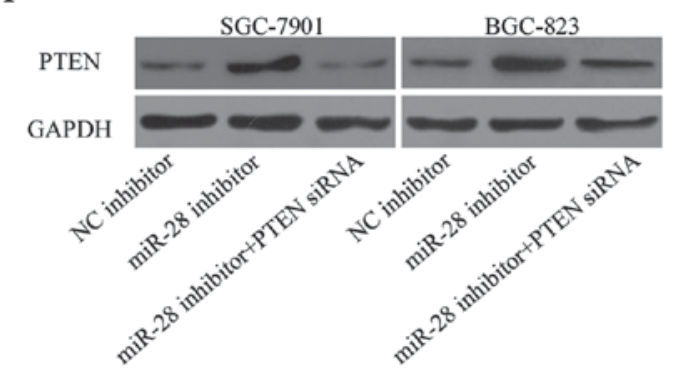

B

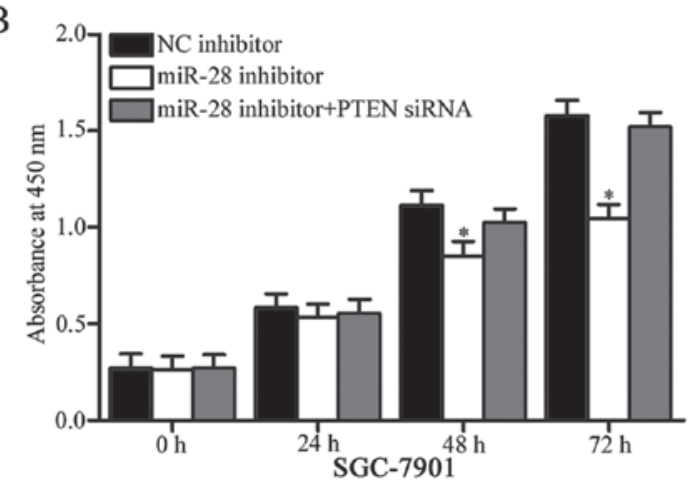

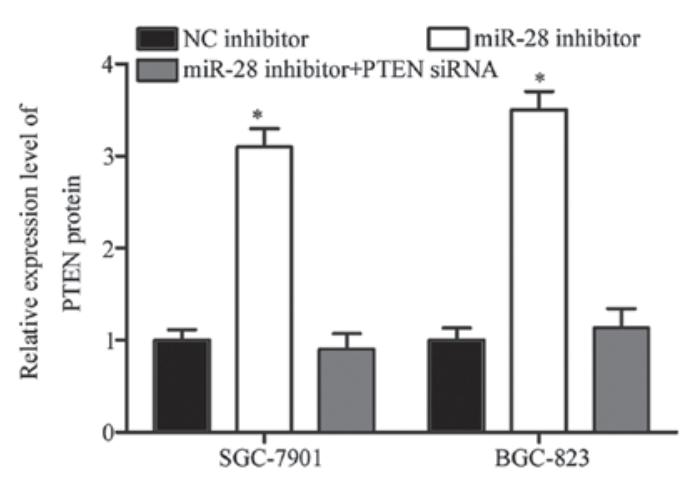

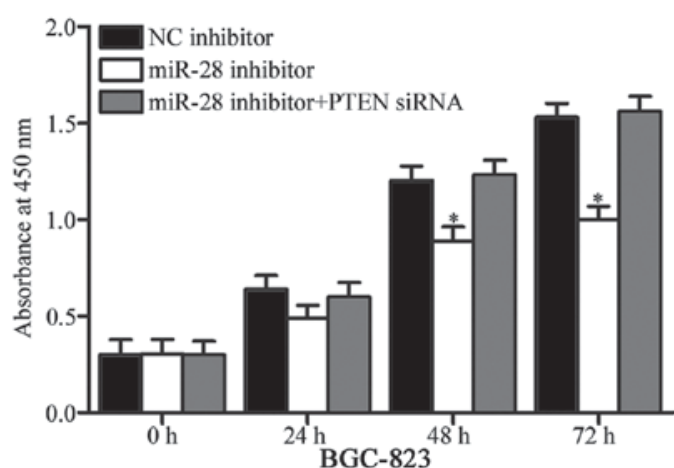

$\mathrm{C}$
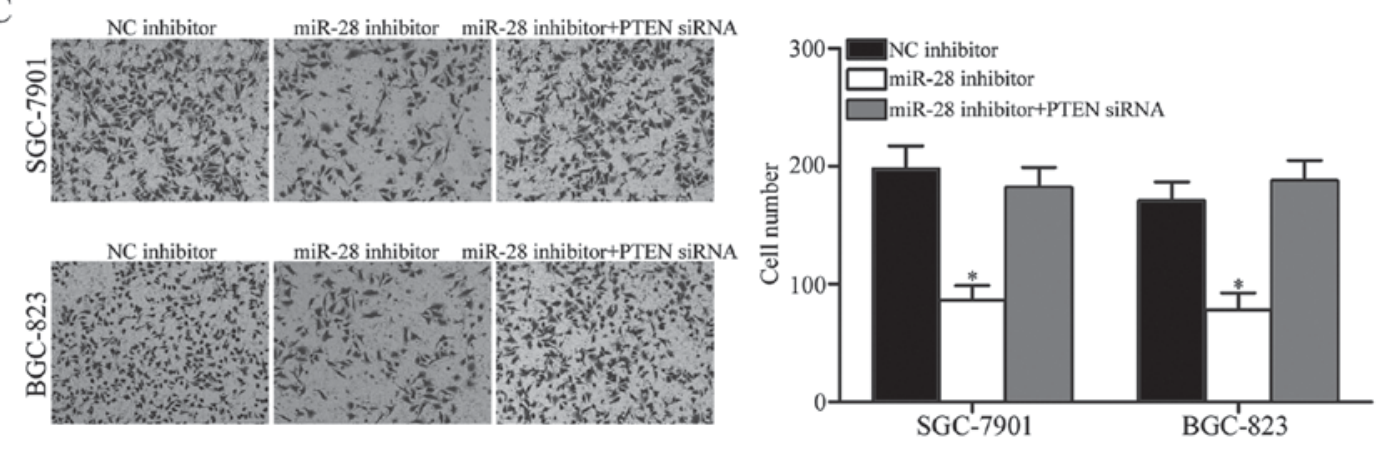

Figure 5. PTEN mediates effects of miR-28 on proliferation and invasion in SGC-7901 and BGC-823 cells. (A) PTEN protein expression was determined by Western blot analysis in SGC-7901 and BGC-823 cells transfected with miR-28 inhibitor with or without PTEN siRNA. ${ }^{*}$ P $<0.05$ compared with NC inhibitor, and miR-28 inhibitor +PTEN siRNA. (B and C) Cell proliferation and invasion were assessed by CCK8 and Matrigel invasion assay in SGC-7901 and BGC-823 cells transfected with miR-28 inhibitor with or without PTEN siRNA. ${ }^{*} \mathrm{P}<0.05$ compared with NC inhibitor, and miR-28 inhibitor $+\mathrm{PTEN}$ siRNA. PTEN, phosphatase and tensin homolog; miR-28, miRNA-28.
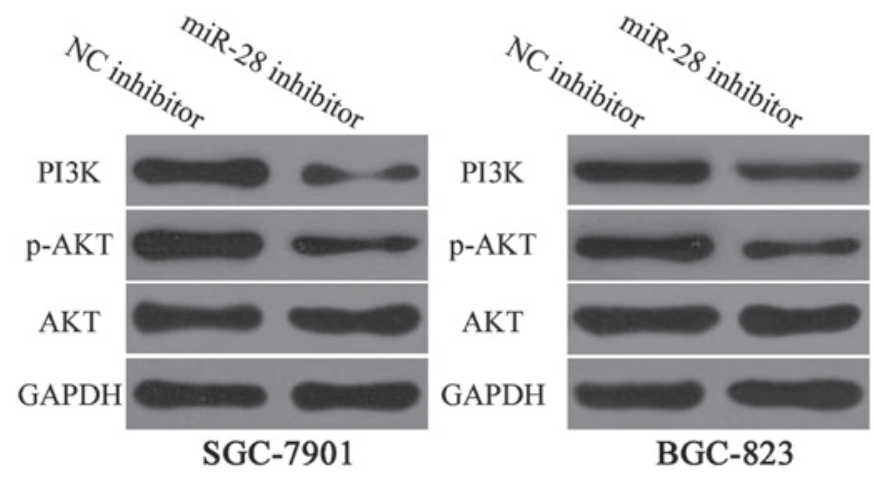

Figure 6. miR-28 underexpression regulates PTEN/PI3K/AKT signalling in gastric cancer cells. SGC-7901 and BGC-823 cells were treated with miR-28 inhibitor or NC inhibitor. Western blot analysis was performed to PI3K, AKT and p-AKT expression. PTEN, phosphatase and tensin homolog; miR-28, miRNA-28.

Recent studies showed that miR-28 expression is deregulated in several human cancers. For example, miR-28 is lowly expressed in hepatocellular carcinoma. Low miR-28 level was correlated with tumour metastasis, recurrence and poor survival of patients with hepatocellular carcinoma (30). Downregulation of miR-28 was also observed in colorectal cancer (18), renal cell carcinoma (19) and B-cell lymphoma (20). However, miR-28 expression is upregulated in ovarian cancer tissues (31). These conflicting findings suggested tissue-specific expression of miR-28.

Tumour-suppressing roles of miR-28 were studied in multiple kinds of human cancer. For example, Zhou et al reported that miR-28 underexpression promoted tumour growth and metastasis of hepatocellular carcinoma in vivo (30). Almeida et al showed that upregulation of miR-28 inhibited colorectal cancer cell proliferation, migration and invasion (18). A study by Wang et al revealed that miR-28 suppressed cell proliferation and migration of renal cell carcinoma in vitro (19). Another study by Schneider et al demonstrated that resumption expression of miR-28 diminished cell proliferation and clonogenic properties of B-cell lymphoma cells (20). miR-28 
was proven to serve as an oncogene in ovarian cancer by regulation of cell proliferation, cell cycle, apoptosis, colony forming and motility. These contradicting findings indicated that miR-28 acts as a tumour suppressor in certain cancers and an oncogene in others.

Several targets of miR-28 were validated; these targets include interleukin-34 (30), insulin-like growth factor-1 (32) in hepatocellular carcinoma, Ras-related protein Rap-1b in renal cell carcinoma (19), MAD2L1 (20) and BCL2 associated athano-gene 1 (20) in B-cell lymphoma and Nedd4-binding partner-1 (31) in ovarian cancer. In our study, PTEN was identified as a novel direct target of miR-28 in gastric cancer. Firstly, bioinformatics analysis predicted that PTEN gene contained a miR-28 seed match at the 3'-UTR of PTEN. Luciferase reporter assay confirmed that zinc finger E-box-binding homeobox 1 directly targeted the 3'-UTR of PTEN gene. Subsequent RT-qPCR and western blot analysis indicated negative regulatory effects of miR-28 on PTEN expression at both mRNA and protein levels. PTEN was also highly expressed in gastric cancer tissues and negatively correlated with miR-28 expression level. PTEN knockdown restored functional roles of miR-28 in gastric cancer cells. Collectively, these data demonstrated that PTEN is a direct and functional downstream target of miR-28 in gastric cancer.

PTEN, located in 10q23.3, is a well-known tumour suppressor (33). Emerging evidence revealed reduced expression levels of PTEN in various human cancers, such as bladder cancer (34), colorectal cancer (35), glioma (36), lung cancer and prostate cancer (37). PTEN deletion was reported to be correlated with aggressive tumour phenotype and adverse prognosis in human cancers (38-40). Accumulated evidence confirmed roles of PTEN in biological processes, such as cell proliferation, cell cycle, apoptosis, migration, invasion, metastasis, metabolism, differentiation, transcription and translation, through inhibition of multiple cell signalling pathways (41-43). In gastric cancer, PTEN is downregulated and negatively correlated with lymph node metastasis, invasion depth, growth pattern, histological classification and age of gastric cancer patients $(22,23,44)$. Functional assays indicated tumour suppressive roles for PTEN in cell apoptosis, cell cycle arrest, proliferation, invasion and metastasis in gastric cancer cells (45-47). Considering importance and role of PTEN in gastric cancer, this homolog may be developed as a therapeutic target for patients with gastric cancer.

In conclusion, miR-28 is upregulated in gastric cancer and plays oncogenic roles through regulation of PTEN/PI3K/AKT signalling pathway. Therefore, this research proposes that miR-28 can be targeted for development of novel treatment for gastric cancer in the future. In following experiments, we will explore the effect of miR-28 on gastric cancer cell proliferation and invasion in vivo.

\section{References}

1. Torre LA, Bray F, Siegel RL, Ferlay J, Lortet-Tieulent J and Jemal A: Global cancer statistics, 2012. CA Cancer J Clin 65: 87-108, 2015.

2. Ferro A, Peleteiro B, Malvezzi M, Bosetti C, Bertuccio P, Levi F, Negri E, La Vecchia C and Lunet N: Worldwide trends in gastric cancer mortality (1980-2011), with predictions to 2015, and incidence by subtype. Eur J Cancer 50: 1330-1344, 2014.
3. Chen W, Zheng R, Baade PD, Zhang S, Zeng H, Bray F, Jemal A, Yu XQ and He J: Cancer statistics in China, 2015. CA Cancer J Clin 66: 115-132, 2016.

4. Kato $\mathrm{M}$ and Asaka M: Recent knowledge of the relationship between Helicobacter pylori and gastric cancer and recent progress of gastroendoscopic diagnosis and treatment for gastric cancer. Jpn J Clin Oncol 40: 828-837, 2010.

5. Li L, Ying XJ, Sun TT, Yi K, Tian HL, Sun R, Tian JH and Yang KH: Overview of methodological quality of systematic reviews about gastric cancer risk and protective factors. Asian Pac J Cancer Prev 13: 2069-2079, 2012.

6. Moon YW, Jeung HC, Rha SY, Yoo NC, Roh JK, Noh SH, Kim BS and Chung HC: Changing patterns of prognosticators during 15-year follow-up of advanced gastric cancer after radical gastrectomy and adjuvant chemotherapy: A 15-year follow-up study at a single korean institute. Ann Surg Oncol 14: 2730-2737, 2007.

7. MaranoL,Polom K,Patriti A,RovielloG,FalcoG,Stracqualursi A, De Luca R, Petrioli R, Martinotti M, Generali D, et al: Surgical management of advanced gastric cancer: An evolving issue. Eur J Surg Oncol 42: 18-27, 2016.

8. Markar SR, Karthikesalingam A, Jackson D and Hanna GB: Long-term survival after gastrectomy for cancer in randomized, controlled oncological trials: Comparison between West and East. Ann Surg Oncol 20: 2328-2338, 2013.

9. Orditura M, Galizia G, Sforza V, Gambardella V, Fabozzi A, Laterza MM, Andreozzi F, Ventriglia J, Savastano B, Mabilia A, et al: Treatment of gastric cancer. World J Gastroenterol 20: 1635-1649, 2014

10. Yu J, Wang R, Chen J, Wu J, Dang Z, Zhang Q and Li B: miR-340 inhibits proliferation and induces apoptosis in gastric cancer cell line SGC-7901, Possibly via the AKT Pathway. Med Sci Monit 23: 71-77, 2017.

11. He L and Hannon GJ: MicroRNAs: Small RNAs with a big role in gene regulation. Nat Rev Genet 5: 522-531, 2004.

12. Bartel DP: MicroRNAs: Genomics, biogenesis, mechanism and function. Cell 116: 281-297, 2004.

13. Shukla GC, Singh J and Barik S: MicroRNAs: Processing, maturation, target recognition and regulatory functions. Mol Cell Pharmacol 3: 83-92, 2011.

14. Garzon R, Calin GA and Croce CM: MicroRNAs in cancer. Annu Rev Med 60: 167-179, 2009.

15. Garzon R and Marcucci G: Potential of microRNAs for cancer diagnostics, prognostication and therapy. Curr Opin Oncol 24: 655-659, 2012

16. Wu D, Niu X, Pan H, Zhou Y, Zhang Z, Qu P and Zhou J: Tumor-suppressing effects of microRNA-429 in human renal cell carcinoma via the downregulation of Sp1. Oncol Lett 12: 2906-2911, 2016

17. Shrestha S, Hsu SD, Huang WY, Huang HY, Chen W, Weng SL and Huang HD: A systematic review of microRNA expression profiling studies in human gastric cancer. Cancer Med 3: 878-888, 2014.

18. Almeida MI, Nicoloso MS, Zeng L, Ivan C, Spizzo R, Gafà R, Xiao L, Zhang X, Vannini I, Fanini F, et al: Strand-specific miR-28-5p and miR-28-3p have distinct effects in colorectal cancer cells. Gastroenterology 142: 886-896.e9, 2012.

19. Wang C, Wu C, Yang Q, Ding M, Zhong J, Zhang CY, Ge J, Wang $\mathrm{J}$ and Zhang C: $\mathrm{miR}-28-5 \mathrm{p}$ acts as a tumor suppressor in renal cell carcinoma for multiple antitumor effects by targeting RAP1B. Oncotarget 7: 73888-73902, 2016.

20. Schneider C, Setty M, Holmes AB, Maute RL, Leslie CS, Mussolin L, Rosolen A, Dalla-Favera R and Basso K: MicroRNA 28 controls cell proliferation and is down-regulated in B-cell lymphomas. Proc Natl Acad Sci USA 111: 8185-8190, 2014.

21. Livak KJ and Schmittgen TD: Analysis of relative gene expression data using real-time quantitative PCR and the 2(-Delta Delta C(T)) method. Methods 25: 402-408, 2001.

22. Fei G, Ebert MP, Mawrin C, Leodolter A, Schmidt N, Dietzmann K and Malfertheiner P: Reduced PTEN expression in gastric cancer and in the gastric mucosa of gastric cancer relatives. Eur J Gastroenterol Hepatol 14: 297-303, 2002.

23. Zheng HC, Li YL, Sun JM, Yang XF, Li XH, Jiang WG, Zhang YC and Xin Y: Growth, invasion, metastasis, differentiation, angiogenesis and apoptosis of gastric cancer regulated by expression of PTEN encoding products. World J Gastroenterol 9: 1662-1666, 2003.

24. Xu WT, Yang Z and Lu NH: Roles of PTEN (phosphatase and tensin homolog) in gastric cancer development and progression. Asian Pac J Cancer Prev 15: 17-24, 2014. 
25. Jing X, Cheng W, Wang S, Li P and He L: Resveratrol induces cell cycle arrest in human gastric cancer MGC803 cells via the PTEN-regulated PI3K/Akt signaling pathway. Oncol Rep 35: 472-478, 2016

26. Wang SQ, Wang C, Chang LM, Zhou KR, Wang JW, Ke Y, Yang DX, Shi HG, Wang R, Shi XL, et al: Geridonin and paclitaxel act synergistically to inhibit the proliferation of gastric cancer cells through ROS-mediated regulation of the PTEN/PI3K/Akt pathway. Oncotarget 7: 72990-73002, 2016.

27. Adams BD, Kasinski AL and Slack FJ: Aberrant regulation and function of microRNAs in cancer. Curr Biol 24: R762-R776, 2014.

28. Iorio MV and Croce CM: MicroRNA dysregulation in cancer: Diagnostics, monitoring and therapeutics. A comprehensive review. EMBO Mol Med 4: 143-159, 2012.

29. Mirnezami AH, Pickard K, Zhang L, Primrose JN and Packham G: MicroRNAs: Key players in carcinogenesis and novel therapeutic targets. Eur J Surg Oncol 35: 339-347, 2009.

30. Zhou SL, Hu ZQ, Zhou ZJ, Dai Z, Wang Z, Cao Y, Fan J, Huang XW and Zhou J: miR-28-5p-IL-34-macrophage feedback loop modulates hepatocellular carcinoma metastasis. Hepatology 63: 1560-1575, 2016.

31. Xu J, Jiang N, Shi H, Zhao S, Yao S and Shen H: (Corrigendum) miR-28-5p promotes the development and progression of ovarian cancer through inhibition of N4BP1. Int J Oncol 50 2236, 2017.

32. Shi $X$ and Teng F: Down-regulated miR-28-5p in human hepatocellular carcinoma correlated with tumor proliferation and migration by targeting insulin-like growth factor-1 (IGF-1). Mol Cell Biochem 408: 283-293, 2015.

33. Maehama T and Dixon JE: The tumor suppressor,PTEN/MMAC1, dephosphorylates the lipid second messenger, phosphatidylinositol 3,4,5-trisphosphate. J Biol Chem 273: 13375-13378, 1998

34. Tanaka M, Koul D, Davies MA, Liebert M, Steck PA and Grossman HB: MMAC1/PTEN inhibits cell growth and induces chemosensitivity to doxorubicin in human bladder cancer cells. Oncogene 19: 5406-5412, 2000.

35. Sawai H, Yasuda A, Ochi N, Ma J, Matsuo Y, Wakasugi T, Takahashi H, Funahashi H, Sato M and Takeyama H: Loss of PTEN expression is associated with colorectal cancer liver metastasis and poor patient survival. BMC Gastroenterol 8: 56, 2008

36. Ermoian RP, Furniss CS, Lamborn KR, Basila D, Berger MS, Gottschalk AR, Nicholas MK, Stokoe D and Haas-Kogan DA Dysregulation of PTEN and protein kinase B is associated with glioma histology and patient survival. Clin Cancer Res 8: 1100-1106, 2002.
37. Mithal P, Allott E, Gerber L, Reid J, Welbourn W, Tikishvili E, Park J, Younus A, Sangale Z, Lanchbury JS, et al: PTEN loss in biopsy tissue predicts poor clinical outcomes in prostate cancer. Int J Urol 21: 1209-1214, 2014.

38. Cordes I, Kluth M, Zygis D, Rink M, Chun F, Eichelberg C, Dahlem R, Fisch M, Höppner W, Wagner W, et al: PTEN deletions are related to disease progression and unfavourable prognosis in early bladder cancer. Histopathology 63: 670-677, 2013.

39. Wise HM, Hermida MA and Leslie NR: Prostate cancer, PI3K, PTEN and prognosis. Clin Sci (Lond) 131: 197-210, 2017.

40. Tao J, Xiong J, Li T, Yang Z, Li X, Li K, Wu H and Wang C: Correlation between protein expression of PTEN in human pancreatic cancer and the proliferation, infiltration, metastasis and prognosis. J Huazhong Univ Sci Technolog Med Sci 26: 444-447, 2006

41. Stambolic V, Suzuki A, de la Pompa JL, Brothers GM, Mirtsos C, Sasaki T, Ruland J, Penninger JM, Siderovski DP and Mak TW: Negative regulation of PKB/Akt-dependent cell survival by the tumor suppressor PTEN. Cell 95: 29-39, 1998.

42. Kohnoh T, Hashimoto N, Ando A, Sakamoto K, Miyazaki S, Aoyama D, Kusunose M, Kimura M, Omote N, Imaizumi K, et al: Hypoxia-induced modulation of PTEN activity and EMT phenotypes in lung cancers. Cancer Cell Int 16: 33, 2016.

43. Nogueira C, Kim KH, Sung H, Paraiso KH, Dannenberg JH, Bosenberg M, Chin L and Kim M: Cooperative interactions of PTEN deficiency and RAS activation in melanoma metastasis. Oncogene 29: 6222-6232, 2010.

44. Zhou YJ, Xiong YX, Wu XT, Shi D, Fan W, Zhou T, Li YC and Huang X: Inactivation of PTEN is associated with increased angiogenesis and VEGF overexpression in gastric cancer. World J Gastroenterol 10: 3225-3229, 2004.

45. Zheng T, Meng X, Wang J, Chen X, Yin D, Liang Y, Song X, Pan S, Jiang H and Liu L: PTEN- and p53-mediated apoptosis and cell cycle arrest by FTY720 in gastric cancer cells and nude mice. J Cell Biochem 111: 218-228, 2010.

46. Zhang LL, Liu J, Lei S, Zhang J, Zhou W and Yu HG: PTEN inhibits the invasion and metastasis of gastric cancer via downregulation of FAK expression. Cell Signal 26: 1011-1020, 2014.

47. He RF, Hu ZL and Wen JF: Biological implication of PTEN gene expression in human gastric cancer and related molecular mechanisms. Zhonghua Bing Li Xue Za Zhi 36: 324-328, 2007 (In Chinese). 